\title{
¿Desde qué perspectiva diseñamos nuestro huerto? Aproximación a la Agroecología desde la formación inicial del profesorado
}

\author{
Lourdes Aragón \\ Dpto. de Didáctica, Área de Didáctica de CC. Experimentales. Facultad de Ciencias de la \\ Educación. Universidad de Cádiz. España. lourdes.aragon@uca.es \\ ORCID: https://orcid.org/0000-0003-3062-9734
}

[Recibido: 2 Julio 2019. Revisado: 21 Septiembre 2019. Aceptado: 5 Noviembre 2019]

\begin{abstract}
Resumen: En el presente artículo se describe una propuesta didáctica llevada a cabo desde la formación inicial del profesorado en la asignatura de Educación Ambiental en Infantil en el 4oㅜ curso del Grado en Educación Infantil. En esta propuesta los estudiantes deben posicionarse, a través de diferentes fuentes bibliográficas, en un marco de referencia sobre el que construir y diseñar nuestro huerto: agricultura convencional, agroecología y permacultura. Dicho marco servirá además para organizar y planificar acciones futuras en el espacio del huerto. De 38 estudiantes, el 54,1\% se posicionó en la agroecología como mejor marco teórico respecto al $43,2 \%$ que optó por la permacultura. Solo un alumno eligió la agricultura convencional. Respecto a los 9 equipos de trabajo, 6 decidieron optar por la agroecología y 3 por la permacultura. Finalmente, en función de la decisión tomada por el grupo clase, se acordó realizar en el huerto diversas acciones desde la visión agroecológica: uso de semillas y plantones ecológicos, diversidad y rotación de cultivos, asociación entre plantas y preparación de biopreparados para prevenir plagas y enfermedades. Estas fueron llevadas a cabo en las siguientes sesiones prácticas del huerto.
\end{abstract}

Palabras clave: Huerto, Formación Inicial de profesores, Agroecología, Permacultura.

Which perspective is designing our garden? Agroecology approaches from Initial Teacher Training

Abstract: This paper presents a didactic proposal based on initial teacher training in the topic Environmental Education for $4^{\text {th }}$ course of Degree in Early Childhood Education Teacher Training. In this proposal the students are asked to position themselves, using diverse bibliography sources about garden design, covering approaches such as: conventional agriculture, agroecology and permaculture. This framework was used to organise, and plan future actions taken into account for garden design. Results showed that from a total of 38 students, $54,1 \%$ were positioned in agroecology theoretical approach while $43,2 \%$ of the students opted for permaculture approach. Furthermore, from 9 proposed work teams, 6 selected agroecology as the best option while 3 groups preferred permaculture frameworks. Finally, based on the decisions made by the students, it was developed a garden following action from an agroecological approach. In this sense the garden design was created using ecological seeds and seedlings, diversity and crop rotation as well as the mix plants and biopreparations to prevent pests and diseases. These tasks were accomplished in practical sessions during the garden design.

Keywords: Garden, Initial Teacher Training, Agroecology, Permaculture.

Para citar este artículo: Aragón, L. (2019) ¿Desde qué perspectiva diseñamos nuestro huerto?. Aproximación a la Agroecología desde la formación inicial del profesorado. Revista de Educación Ambiental y Sostenibilidad 1(2), 2201. doi: 10.25267/Rev_educ_ambient_sostenibilidad.2019.v1.i2.2201 


\section{Introducción}

Cada vez son más los centros escolares que emplean el huerto como recurso didáctico en las diferentes etapas educativas. Un recurso que, desde hace décadas, se integra como un espacio más de aprendizaje en el centro. El huerto escolar posee un elevado potencial didáctico para trabajar en torno a los contenidos escolares y que desde hace algún tiempo, se viene empleando para alcanzar finalidades didácticas más ambiciosas dirigidas a la transformación social y a formar a futuros ciudadanos y ciudadanas comprometidos con su medio ambiente (Rodríguez-Marín, FernándezArroyo, Puig-Gutiérrez y García, 2017). A nivel nacional, muchos huertos escolares funcionan a modo de red; por ejemplo, la Red de Huertos Escolares Sostenibles del Ayuntamiento de Madrid, formada desde el curso 2015-2016 y que integra actualmente 165 centros (Estrella, Del Valle y Jiménez, 2017). En otras comunidades como el País Vasco o Canarias, que cuentan respectivamente con alrededor de 350 y 280 centros que trabajan en torno a este recurso. Datos recientes estiman que aproximadamente, 1.000 .000 de escolares trabajan con huertos escolares (Estrella, 2018).

Ante esta situación, los diversos profesionales que utilizamos el Huerto Ecológico Universitario (HEU en adelante) como recurso y contexto de aprendizaje en la formación inicial del profesorado, no podemos permanecer ajenos, sino más bien, sumarnos a dichas iniciativas, promover acciones y propuestas didácticas encaminadas a crear puentes de colaboración entre universidades y escuelas (Aragón y Cruz, 2016a). Consideramos que desde la formación inicial del profesorado debemos asumir nuestra responsabilidad a la hora de trabajar con los futuros maestros este recurso para que en su futura práctica docente integren metodologías y estrategias con enfoques adecuados, reduciendo la improvisación, y siendo más conscientes del potencial que posee el huerto escolar para trabajar todas las áreas del curriculum. Kaufman (1995) ya exponía en su trabajo el enorme potencial didáctico que posee el huerto en la etapa de infantil, permitiendo conectar y relacionar todas las áreas de conocimiento a través de una metodología investigativa que implica a estudiantes y docentes. El huerto ofrece la oportunidad de aprender a respetar tiempos, es decir, acercar a los niños (y docentes) a la producción de los alimentos, asumiendo que la naturaleza posee sus propios ritmos, cíclicos, y más lentos que los nuestros. Contribuye a que niños y niñas de edades tempranas, construyan su concepción del mundo, de sí mismo y de los otros, e incluso empezar a conocer sus emociones, dado que el huerto es un contexto que favorece la observación, exploración, experimentación e interrelación de los elementos que conforman el medio que les rodea, sobre todo a través del juego y la imitación (Martínez-Madrid y Eugenio, 2016).

En la etapa de primaria, el huerto se configura también como un contexto idóneo para desarrollar la competencia ecociudadana del alumnado. Un espacio que permite trabajar desde otros marcos de referencia como es la agroecología escolar (Llerena y Espinet, 2017). Esta perspectiva se considera más idónea para lograr la transformación y cambio social, ya que favorece entornos escolares más democráticos y participativos, que permite organizar los contenidos de otra manera (Rekondo, Espinet y Llerena, 2015). El huerto es entendido como un espacio que funciona de forma distinta a otros espacios escolares. Es considerado como un 
producto híbrido, en torno al cual se generan tensiones y discusiones entre los diferentes agentes comunitarios que se implican en él y que, en el mejor de los casos, permite generar nuevos discursos y nuevas prácticas educativas (Amat, 2013). Sin embargo, integrar el huerto en el entorno escolar no es tarea fácil. Según Ceballos (2017) aún son minoritarios los centros escolares que emplean el huerto como recurso para estimular en sus alumnos estrategias centradas en la indagación siendo el objetivo didáctico más frecuente el de facilitar que el alumnado ponga en práctica los contenidos teóricos vistos en el aula.

El uso de HEU también vive en estos momentos una enorme fertilidad didáctica. Esta proliferación ha propiciado la creación, en 2015, de una asociación de ámbito estatal denominada Red Universidades Cultivadas (RUC), constituida por un grupo interdisciplinar de personas vinculadas a la universidad y a otras entidades sociales, que incorporan los huertos con finalidades didácticas a su actividad docente o investigadora desde diferentes áreas de conocimiento (Eugenio y Aragón, 2016).

Desde la formación inicial del profesorado existe una larga trayectoria en este sentido y algunas universidades emplean el huerto como espacios socioeducativos transversales en los que conviven futuros docentes y escuelas; es el caso de la Red de Huertos Comunitarios-Escolares de la Universidad de Salamanca (Barrón y Muñoz, 2015). En otras universidades, el HEU se integra en asignaturas en los Grados en Educación Infantil y Primaria vinculadas a las ciencias experimentales y a la educación ambiental. Conviven igualmente perspectivas distintas y metodologías educativas diversas (Ceballos, Escobar y Vílchez, 2014; Eugenio, 2016; Aragón y Cruz, 2016b) así como enfoques diferentes para abordar los problemas socioambientales (Aragón, Jiménez-Tenorio y Oliva, 2015) y desde la sostenibilidad (Eugenio, Zuazagoitia y Ruiz-González, 2018). Sin embargo, los futuros docentes presentan ciertas dificultades a la hora de diseñar propuestas didácticas para trabajar problemas socioambientales en torno al huerto. Aunque vivencien estrategias de corte investigativo se detectan ciertas carencias en el marco didáctico. Se tiende a polarizar los objetivos y contenidos conceptuales frente a los procedimentales, y la metodología basada en la investigación es casi inexistente en sus propuestas (Aragón, 2017).

Ante las diversas dificultades que aún se detectan en la formación inicial del profesorado y de acuerdo con Ceballos (2017), debemos seguir con nuestra labor de formar a los futuros docentes en este campo. En este sentido, el presente artículo pretende, por un lado, poner en valor el uso del huerto como recurso didáctico para la formación inicial del profesorado, en concreto, de futuros maestros de Infantil y, por otro, diseñar una propuesta didáctica con el fin de acercar al alumnado a diferentes modelos agrícolas que den sentido y fundamenten las posibles acciones que llevarán a cabo en el espacio del huerto. Se consensúa con el alumnado la mejor perspectiva teórica para construir un huerto ecológico en el espacio disponible para ello. Posteriormente, los estudiantes propondrán, de acuerdo al marco consensuado, las primeras acciones para realizar en el huerto. Se espera que el alumnado sea más consciente de los distintos planteamientos teóricos que existen a la hora de poner en marcha un huerto (agricultura convencional, agroeocología o permacultura), de forma que puedan ser trasladadas a sus prácticas didácticas futuras. Se pretende además, a través de esta propuesta, empoderar al alumnado y fomentar su visión 
crítica ante el consumo de alimentos, lo cual puede llevar asociados cambios de actitud y compromiso hacia el medio.

\section{Posibles perspectivas: agricultura convencional, agroecología y permacultura}

\section{La agricultura convencional: la revolución verde}

La agricultura convencional, también denominada revolución verde, tiene lugar en la década de los años 50 a partir de la postguerra mundial e iniciada por países capitalistas (González, 1984). Nace con la finalidad de lograr elevadas tasas de productividad agrícola y con una base tecnológica importante, basada principalmente en la selección genética de nuevas variedades de cultivo (Ceccon, 2008). Según Bello, Jordá y Tello (2010), se caracteriza por el uso masivo de agroquímicos (herbicidas, pesticidas, fertilizantes químicos) y maquinaria pesada. Estas prácticas agrarias reduccionistas han sido consideradas una de las causas que han conducido a la situación de crisis ambiental y que, en los años 70, provocaron una respuesta masiva iniciada por los movimientos ecologistas. Esta perspectiva posee importantes implicaciones sociales y económicas, dado que la agricultura convencional ha estado dirigida a crear nuevos monopolios. En los años 90 tiene lugar una segunda revolución verde que integra la biotecnología con la ingeniería genética, con el principal objetivo de crear organismos genéticamente modificados, denominados transgénicos (Ceccon, 2008). Ambas revoluciones han sido justificadas como una solución al problema del hambre, principalmente de los países de Asia, aumentando la rentabilidad productiva del terreno sobre todo de tres cultivos concretos: trigo, maíz y arroz (Barrera, 2011). Los cambios tecnológicos introducidos por la agricultura convencional, concebidos como innovaciones, se refuerzan mutuamente, dado que la mecanización agraria de un cultivo se puede acompañar del uso de nuevas variedades de plantas que, a su vez, dependen estrechamente del uso generalizado de productos químicos (González, 1984). La agricultura convencional no solo produce deforestación, también ha provocado una aceleración de los procesos de erosión, desertificación, reducción de la biodiversidad e incremento de las plagas y enfermedades (Laurín, Llosá, Gonzálvez y Porcuna, 2006). La agricultura representa el uso de una enorme proporción de tierra empleada por el hombre y supone una importante fuente de emisión de gases que contribuyen al efecto invernadero. Las prácticas agrícolas intensivas, como la cría de ganado, el cultivo de arroz y el uso de fertilizantes emiten más del $50 \%$ del metano y gran parte del óxido nitroso proveniente de actividades humanas (García, Laurín, Llosá, Gonzálvez, Sanz y Porcuna, 2006). Por otro lado, el uso indiscriminado de pesticidas acarrea diversos problemas, de los que gran parte tienen que ver con la contaminación del suelo y la contaminación de los cursos del agua, lo cual conlleva, a su vez, daños sobre la flora y la fauna acuática y terrestre o la resistencia a la plagas, entre otros (Espluga, 2001). Se trata de un modelo agrícola con importantes consecuencias para la naturaleza y para la sociedad, puesto que, según Altieri (2013) amenaza la seguridad alimentaria de millones de personas dada a su alta dependencia de los hidrocarburos, que provoca que los costes de los alimentos crezcan de una manera desproporcionada. 


\section{La perspectiva agroecológica}

Las prácticas agroproductivas convencionales han propiciado una profunda situación de crisis ecológica a nivel planetario, a la vez que son consideradas como socialmente desiguales por beneficiar únicamente a un pequeña parte de la población, y causar que los agricultores carezcan de autonomía y sean dependientes de semillas o de los propios mercados (Martínez, 2002). A esta situación se le suman, además, otros problemas socioambientales, que según Altieri y Nicholls (2000) tienen que ver tanto con el crecimiento demográfico como con el incremento de la demanda económica y social que ya se manifestaba hace dos décadas. Estos autores ya contemplaban dos desafíos cruciales, vigentes a día de hoy, a los que el mundo académico y el mundo del desarrollo debían y deben hacer frente. Por un lado, es necesario incrementar la producción agrícola a nivel regional entre un 30 y-40\%, sin agravar la situación actual de degradación ambiental, y, por otro, lograr un acceso más igualitario de la población, no solo a los alimentos, sino también a los recursos necesarios para producirlos. La agroecología surge así como un ámbito científico prioritario que permite la conversión de los sistemas convencionales de producción, ya comentados en el apartado anterior, a sistemas diversificados y autosuficientes (Altieri y Nicholls, 2017).

La agroecología se define como una disciplina científica enfocada hacia el estudio de la agricultura desde una visión ecológica, con el objeto de analizar los procesos agrícolas desde una manera más amplia y holística. Desde esta perspectiva teórica, los ecosistemas agrícolas (agroecosistemas) son considerados como unidades fundamentales de estudio que se analizan e investigan como un todo, atendiendo a los ciclos minerales, las transformaciones de energía, los procesos biológicos y las relaciones socioeconómicas (Altieri y Nicholls, 2000). De acuerdo a Griffon (2008), manejar holísticamente los agroecosistemas pasa por traspasar las prácticas reduccionistas predominantes de las ciencias agrícolas convencionales. La agroecología no solo abarca la producción de alimentos, sino que, como indica Martínez (2002), se extiende a los aspectos culturales, sociales y económicos que se relacionan e influyen en la producción: elaboración, transformación, comercialización, consumo y tratamiento como residuo. Es decir, todo lo que conforma el sistema alimentario. Según Llerena y Espinet (2017) cada uno de esos procesos debe ser abordado desde las distintas dimensiones que componen la agroecología: ecológico-ambiental, tecnológico, socioeconómico, cultural y político. Asimismo, dichas dimensiones abarcan una amplia diversidad de conceptos y características; Llerena y Espinet (2017), proponen algunos: ecojusticia, participación, soberanía, perspectiva de género, etc., que muestran el enorme carácter transdiciplinario que contempla esta disciplina.

En la práctica, la perspectiva agroecológica se sustenta en dos principios básicos que deben ser atendidos en el diseño de los agroecosistemas. Altieri y Nicholls (2017, p. 2) exponen que estos deben ser:

1) el manejo orgánico del suelo, para lo cual es necesario incrementar la cantidad de materia orgánica, el reciclaje de nutrientes y aumentar la biota edáfica; 
2) el manejo del hábitat y diversificación a través del uso de policultivos, los cultivos de cobertura, las rotaciones, uso de corredores, etc., con el objetivo de incrementar la fauna benéfica.

No obstante, aunque la agroecología propone alternativas al sistema convencional se tata de una disciplina que continua gestándose y definiéndose, de manera que aun se están revisando sus propias bases epistemológicas (Gómez, Ríos-Osorio y Eschenhagen, 2015).

\section{La permacultura}

El término permacultura significa cultura permanente, un concepto que fue introducido por Bill Mollison y David Holmgren a mediados de los años 70 como alternativa al sistema agrícola convencional. Holmgren (2013) define esta perspectiva como el diseño consciente de paisajes que imitan los patrones y las relaciones de la naturaleza, a la vez que suministra alimento, fibras y energía suficientes para satisfacer las necesidades locales.

La permacultura alude a un sistema sostenible que integra de manera armónica la vivienda, el paisaje, con el objeto de disminuir materiales y minimizar desechos, con importantes repercusiones a la hora de conservar los recursos naturales disponibles (Rodríguez, Hernández, Hernández y Pérez, 2015). De acuerdo con Torres y Soto (2016), la permacultura es un sistema de diseño que busca crear asentamientos comunitarios sostenibles, ecológicamente sanos y viables económicamente configurándose como una alternativa a los problemas socioambientales actuales. Desde la permacultura se toma más conciencia de los límites biofísicos del planeta, en cuanto a la disposición del uso de energía y de los recursos naturales, muy acorde a la corriente decrecentista, centrada en concienciarse de los límites biofísicos, comprender los límites del crecimiento y las prácticas agrícolas que presentan un mayor rendimiento energético (Rodríguez-Marín, Fernández-Aguayo y García, 2015).

Mollison (1979) establece tres principios éticos fundamentales que nutren esta perspectiva:

1) el cuidado de la tierra; usar y manejar de manera responsable los recursos, integrando los ciclos de materia y flujos de energía con una perspectiva a largo plazo;

2) el cuidado de las personas; el componente social, que requiere de proveer a las comunidades al acceso de los recursos necesarios para su existencia, y no solo en lo referente a la alimentación, también a otros ámbitos de su vida como el derecho a una educación, sanidad, vivienda, etc., atendiendo a la capacidad de regeneración que poseen los ecosistemas;

3) establecer límites en cuanto a la población y el consumo; se corresponde al componente económico, y se basa en repartir y compartir los recursos atendiendo a la idea de que vivimos en un planeta finito, con recursos limitados y, por tanto, debemos limitar también nuestro consumo y producción.

La permacultura se percibe como una alternativa a la agricultura convencional a través de una visión holística que trata los problemas agrícolas desde un enfoque integrado. Caballero y Montes (1997) recogen algunos de los objetivos que se promueven desde esta corriente de pensamiento: crear sistemas agrícolas de bajo coste energético y alta productividad, integrando aspectos agrícolas, forestales y 
pecuarios. Y concebir la vivienda como una parte integrada del ciclo ecológico de la propia parcela, buscando el mayor grado de autosuficiencia, empleando para ello, técnicas y tecnologías accesibles, tanto desde una perspectiva económica como tecnológica, para cualquier persona.

\section{Propuesta didáctica: contexto y participantes}

La propuesta didáctica que se describe a continuación se ha realizado en el 4 o curso del Grado en Educación Infantil de la Universidad de Cádiz, desde la asignatura de Educación Ambiental en Infantil (EAI) en el segundo semestre del curso 2018-2019. Se trata de una asignatura optativa de 6 ECTS, con 4 créditos teóricos y 2 prácticos (UCA, 2012). El huerto se emplea fundamentalmente en las sesiones prácticas de hora y media de duración realizadas con los dos subgrupos de estudiantesy a lo largo de las 15 semanas que se estructura el semestre. El trabajo del huerto se comparte con otros espacios de aprendizaje como el aula y el laboratorio. El huerto se sitúa en un patio interior de la propia Facultad, con dimensiones de unos $60 \mathrm{~m}^{2}$ aproximadamente (figura 1).

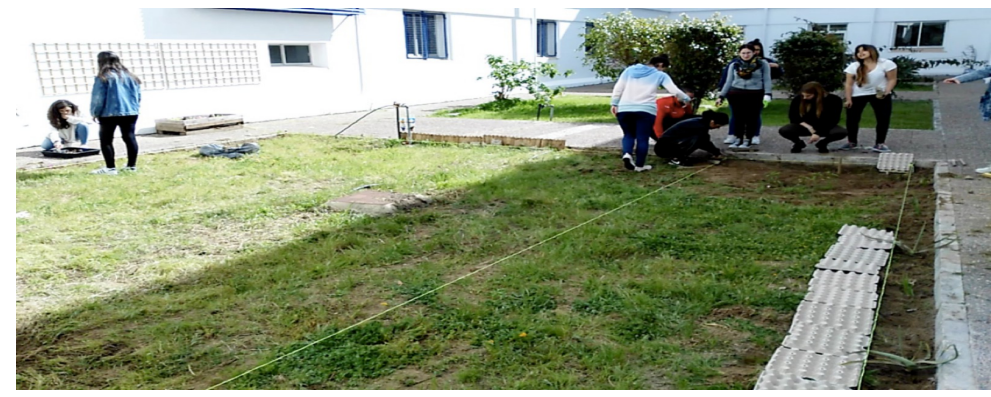

Figura 1. Huerto Ecológico Universitario situado en uno de los patios interiores de la Facultad de Ciencias de la Educación (Universidad de Cádiz).(Fuente: elaboración propia).

En cada curso académico los estudiantes deben diseñar desde cero el huerto, que se encuentran únicamente con plantas adventicias. En este curso, como mejora, se han construido 5 bancales utilizando bloques de hormigón de 40x10x20 cm. El diseño y la organización de dichos bancales se ha realizado a través de varias sesiones prácticas durante la fase final de la asignatura de EAI (figura 2). El número de estudiantes matriculados cada año es variable, dado que se trata de una asignatura optativa. Durante este curso académico, el grupo-clase estuvo formado por 38 estudiantes (5 hombres y 33 mujeres) de edades comprendidas entre los 21 y los 40 años. Los estudiantes se agrupan por equipos de trabajo de entre 3-6 componentes cada uno, con lo que se cuenta con un total de 9 grupos.

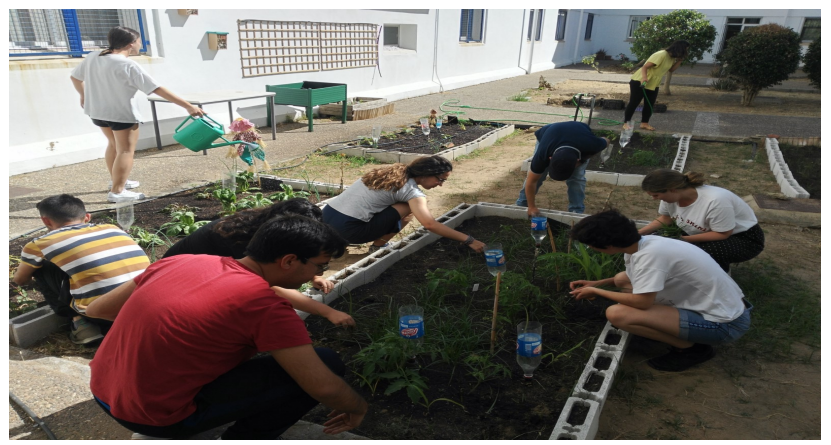

Figura 2. Diseño del HEU a través de bancales en la asignatura de EAI durante el curso 2018-2019. (Fuente: elaboración propia). 
El HEU dispone de otros espacios: zona de aprendizaje, huerto vertical, mesa de cultivo, compostadora, zona de almacenaje y un punto de agua. Cada curso académico se dota con semillas y plantas, algunas ecológicas y otras híbridas, un saco de abono y otro de sustrato cedidos por la Diputación de Cádiz, a través de la inscripción al Programa de Huertos Escolares.

\section{Descripción de la propuesta didáctica}

La propuesta didáctica fue diseñada por la autora de este trabajo, es de corte constructivista y fue implementada a lo largo de seis sesiones al inicio de la asignatura. En la tabla 1 se especifica la organización y secuenciación de las sesiones prácticas, así como los distintos agrupamientos de los estudiantes en cada una de ellas. El objetivo final de la secuencia es diseñar y poner en marcha desde la asignatura un huerto ecológico. Este trabajo se centra en la fase de desarrollo y reestructuración, para ello se describirán a continuación las sesiones 4, 5 y 6, dado que fueron las actividades claves para tratar la pregunta problema: ¿desde qué perspectiva construir nuestro huerto ecológico? Las sesiones anteriores han sido descritas con más detalle en Aragón y Rodríguez-Marín (2019).

Tabla 1. Secuenciación de sesiones prácticas en la asignatura de EAI en el 4o curso del Grado en Educación Infantil durante el curso 2018-2019. (Fuente: elaboración propia).

\begin{tabular}{|c|c|c|c|}
\hline $\begin{array}{l}\text { Fases Propuesta } \\
\text { Didáctica }\end{array}$ & $\begin{array}{l}\text { Semanas } \\
(15 \text { totales })\end{array}$ & Secuencia de actividades & Agrupamientos \\
\hline \multirow{5}{*}{$\begin{array}{l}\text { Fase inicial: } \\
\text { despertar el interés } \\
\text { hacia el tema del } \\
\text { huerto y explicar } \\
\text { ideas previas }\end{array}$} & Sesión 1 & $\begin{array}{l}\text { Act. } 1 \text {. Mesas paneles para explicitar perspectivas de } \\
\text { aprendizaje e interés en torno al huerto ecológico como } \\
\text { recurso de aprendizaje en la asignatura de EAI. }\end{array}$ & $\begin{array}{l}\text { Individual Grupos } \\
\text { aleatorios }\end{array}$ \\
\hline & & Act. 2. ¿Qué modelos mentales sobre huertos tenemos? & Equipos de trabajo \\
\hline & Sesion 2 & Act. 3. Explicitación de ideas previas sobre los modelos & \\
\hline & & iniciales de huerto ecológico. & Gran grupo \\
\hline & Sesión 3 & $\begin{array}{l}\text { Act. 4. ¿Qué elementos existen en nuestro espacio para } \\
\text { diseñar el huerto? }\end{array}$ & Equipos de trabajo \\
\hline $\begin{array}{l}\text { Fase de desarrollo y } \\
\text { reestructuración }\end{array}$ & Sesión 4 & $\begin{array}{l}\text { Act. 5. ¿Desde que marco teórico construir nuestro } \\
\text { huerto ecológico? (I) }\end{array}$ & $\begin{array}{l}\text { Individual Equipos } \\
\text { de trabajo }\end{array}$ \\
\hline \multirow{2}{*}{$\begin{array}{l}\text { Fase final: toma de } \\
\text { decisiones y } \\
\text { establecimiento de } \\
\text { acuerdos para } \\
\text { comenzar la } \\
\text { siguiente pronuesta }\end{array}$} & Sesión 5 & $\begin{array}{l}\text { Act. 6. ¿Desde qué marco teórico construir nuestro } \\
\text { huerto ecológico? (II) }\end{array}$ & Gran grupo \\
\hline & Sesión 6 & $\begin{array}{l}\text { Act. 7. Diseño y organización del espacio para creación } \\
\text { de un huerto ecológico (I). }\end{array}$ & $\begin{array}{l}\text { Equipos de trabajo } \\
\text { Gran grupo }\end{array}$ \\
\hline
\end{tabular}

El objetivo de la propuesta didáctica fue acercar al alumnado a marcos alternativos al sistema convencional de producción, bien desde la agroecología o la permacultura. Ambos igualmente válidos, pero quizás, dada la bibliografía consultada, la agroecología se trata de la alternativa que se considera más apropiada para trabajar con futuros docentes, ya que existe todo un campo teórico emergente, que permite su transposición didáctica a través de la agroecología escolar (Llerena y Espinet, 2017). Esta vincula huertos, educación, escuela, investigación y universidad, e integra el sistema alimentario -incluyendo la producción, el transporte, la trasformación y el tratamiento de residuosa los contenidos curriculares y a la vida diaria de la escuela (Martínez-Madrid y Eugenio, 2016). 
En la sesión 4, la docente planteó la pregunta problema anterior; los alumnos, de manera individual debían elaborar un guión-reflexión proporcionado por la docente. En este se solicitaban cuatro aspectos concretos:

1) extraer los principios básicos que definen cada una de las perspectivas propuestas a través de los documentos bibliográficos aportados por la docente. Para ello, debían atender a tres criterios clave que posteriormente serían empleados para la fase de discusión y debate: la entrada y salida de recursos utilizados, aspectos sociales y económicos (autosuficiencia o dependencia de semillas) e impacto ambiental (espacio de terreno utilizado, consecuencias ambientales y éticas);

2) señalar palabras claves y buscar en el diccionario si no se conoce su significado;

3) decidir qué perspectiva se considera la más idónea para crear un huerto ecológico argumentando dicha elección en base a los criterios anteriores; y

4) describir dos acciones para llevar a cabo en el espacio del huerto acorde a la perspectiva elegida.

Las lecturas proporcionadas al alumnado ofrecían un panorama general e información básica sobre cada una de las perspectivas con el objeto de realizar una primera aproximación a cada una de ellas. Los tres artículos seleccionados fueron:

1) La revolución verde y plaguicidas en el ambiente (lectura 1). Un ensayo donde el autor expone la situación actual en torno a la demanda de alimento con respecto al creciente aumento de la población, y la necesidad de aumentar la producción. Se expone el uso de una agricultura basada en el uso de pesticidas, herbicidas e insecticidas. Se describe de manera muy general en qué consiste la agricultura convencional, incidiendo en las bases de la revolución verde y algunas de las consecuencias con el uso de pesticidas fundamentalmente.

2) ¿Qué es la permacultura? (lectura 2). La autora de este artículo ofrece una definición amplia de la permacultura y sus principios básicos. Se aportan dos ejemplos que permite acercar esta perspectiva desde un espacio amplio como puede ser una finca, pero también desde espacios más reducidos (la ciudad, balcones, terrazas, patios, áticos o jardineras).

3) Agroecología, una alternativa viable (lectura 3). Se ofrece información sobre los principios de la agroecología y los objetivos de esta disciplina, así como los mecanismos para mejorar la inmunidad de los agroecosistemas. Es un documento que, a diferencia de los anteriores, ambos con un carácter más divulgativo, posee un lenguaje más técnico y específico sobre muchos de los aspectos claves para discutir.

Posteriormente, los estudiantes, organizados por equipos de trabajo, debían compartir la información construida en sus informes individuales, explicar sus posiciones y llegar a un acuerdo sobre el marco teórico sobre el que diseñar nuestro huerto ecológico, atendiendo a los tres criterios claves. Asimismo, también debían tomar decisiones respecto a dos actuaciones concretas basadas en el marco teórico de referencia para llevar a cabo en el espacio del huerto. Todo ello se debía registrar en su portafolio grupal. 
La sesión 5 fue utilizada para que los equipos de trabajo expusieran sus posiciones y compartieran sus argumentos en gran grupo. La docente actúo como moderadora registrando en la pizarra el marco teórico elegido por cada equipo de trabajo así como los argumentos empleados atendiendo a los criterios claves. Además, los estudiantes discutieron sobre algunas de las palabras que desconocían y habían buscado, solucionaron dudas y realizaron algunas valoraciones de las lecturas aportadas por la docente. Finalmente, se inició un debate en gran grupo y se procedió a la elección final del marco teórico más idóneo para diseñar nuestro huerto ecológico tras el consenso del grupo clase. La docente focalizó el debate en los criterios claves anteriores, lo que permitió comparar los tres modelos agrícolas proporcionados y cuestionar algunas de sus concepciones y argumentos empleados.

La sesión 6 se inició con una explicación por parte de la docente de los tres marcos teóricos propuestos. Se aportó información complementaria a los textos facilitados para ayudar a comprender y poder profundizar en cada uno de los marcos expuestos y solucionar dudas planteadas. Se introdujeron conceptos claves como soberanía alimentaria, semilla ecológica vs. transgénica, visón holística, empoderamiento o monopolio, presentes en las lectura dadas, ya que muchos estudiantes presentaban dudas al respecto. Finalmente, se confrontaron los modelos acordados por los grupos y sus argumentos empleados. Una vez consensuado el modelo desde el cual diseñar y crear el huerto ecológico, cada grupo expuso dos acciones que debían ser llevadas a cabo en el espacio del huerto, de acuerdo con dicha perspectiva. Se acordaron aquellas que eran más viables y coherentes con el marco elegido y los criterios discutidos.

\section{Resultados de la implementación de la propuesta didáctica}

Para profundizar en los argumentos utilizados por los estudiantes, tanto individualmente como por los equipos de trabajo, se analizaron post-hoc sus producciones de cara a mejorar la propia propuesta didáctica en próximos cursos académicos.

\section{Actividad individual}

En la tabla 2 se muestra un resumen del sistema de categorías emergente empleado para analizar las respuestas individuales de los estudiantes a la hora de posicionarse ante un modelo para diseñar el huerto ecológico.

Tabla 2. Sistema de categorías emergente para analizar respuestas del alumnado en la fase individual de la propuesta didáctica (Fuente: elaboración propia).

\begin{tabular}{llr}
\hline Modelos agrícolas (N=38) & Respuestas de los estudiantes & No(\%) \\
\hline Agricultura convencional (5,3\%) & Productividad & $1(100 \%)$ \\
\hline Agroecología (52,6\%) & Productividad & $11(55 \%)$ \\
& Viabilidad & $6(30 \%)$ \\
& Principios básicos que sustenta la agrocología & $3(15 \%)$ \\
\hline Permacultura (42,1\%) & Principios que sustentan esta corriente & $14(87,5 \%)$ \\
& Productividad & $1(6,25 \%)$ \\
& Otros criterios: fácil comprensión frente a la otra & $1(6,25 \%)$
\end{tabular}

Respecto a las posiciones individuales, el 52,6\% (20) de los estudiantes optaron por la agroecología como la perspectiva más adecuada para aplicar en un huerto 
ecológico. Entre sus respuestas se encuentran argumentos centrados en tres aspectos: la productividad del huerto, la viabilidad y los principios básicos que sustentan esta corriente. Los argumentos más frecuentes son los que aluden a la productividad; 11 de los 20 estudiantes exponen la agroecología como mejor perspectiva dado que logra producir alimentos. Respecto a esta idea se encuentran argumentos del estilo «este modelo es el más acertado para nuestro huerto debido a que a nosotros lo que nos interesa es la producción» (A.5), o bien «ya que es el más sencillo para la producción y el consumo» (A.6).

Otros seis estudiantes aportan argumentos relacionados con la viabilidad y lo comparan con la permacultura. Algunos de ellos contemplan que la permacultura sería la mejor opción, sin embargo, no es alcanzable en el tiempo del que se dispone en la asignatura de EAI. En este sentido, se encuentran argumentos del estilo "la permacultura es demasiado difícil de llevar a cabo..., la agroecología es el modelo intermedio entre la agricultura convencional y la permacultura» (A.7), o «la mejor es la permacultura pero la veo un poco utópica para llevarla a cabo en el huerto» (A.13). Otros dos alumnos consideran que la agroecología es una corriente adaptable al centro escolar, y que supone una opción real. Un bajo número de estudiantes aluden en sus respuestas a argumentos que se relacionen con los principios básicos de esta corriente, principalmente por su capacidad de integrar elementos naturales y humanos de manera más sustentable.

Por otro lado, el 42,7\% (16) de los alumnos eligieron la permacultura. En este caso, entre sus respuestas se denota una visión más holística, dado que 14 de ellos argumentan utilizando los tres principios que sustentan esta perspectiva. En algunas respuestas se observa que son conscientes del trabajo y esfuerzo que conlleva esta perspectiva, sin embargo, las asumen independientemente del tamaño del huerto, y del tiempo disponible para ello. Así, se encuentran respuestas del tipo «la permacultura nos ayudaría a contribuir con la sostenibilidad del planeta, nos podría aportar otra perspectiva para poder trabajar en comunidad y no de forma competitiva, así tomaríamos conciencia de que todos debemos mejorar por la misma causa» (A.23); «Elijo la permacultura porque se enfoca en que el planeta sea más sostenible y eso es lo que debemos conseguir trabajando en nuestro huerto. Además de cuidar la tierra, tenemos que ver nuestro trabajo en el huerto como una forma de vivir, de sentir...» (A.25). Solo dos alumnos aluden a otros aspectos, como son la productividad como objetivo del huerto, y por otro, la dificultad que entraña esta perspectiva con respecto a la agroecología; sin embargo, se decanta por la permacultura debido a que ha entendido mejor el artículo. Los argumentos que sustentan la opinión del alumnado son fundamentales para entender la opción final consensuada por el grupo clase. En la figura 3 se puede observar un cambio en la opinión individual del alumnado al compartir sus ideas con los compañeros dentro del equipo de trabajo.

Se observa que en los grupos en que existe al menos un componente con la opción individual de permacultura pasan a optar por la perspectiva de la agroecología. Esto ocurre en los equipos 2, 4, 8 y 9. Probablemente los alumnos que apoyan la agroecología han sido capaces de convencer y argumentar sus posiciones frente a la permacultura, sobre todo aludiendo a algunos de los tres argumentos predominantes expuestos anteriormente. Por otro lado, se da un caso en que ocurre lo contrario; un grupo formado por un componente que apoya la agroecología se decanta finalmente por la permacultura. La visión holística quizás resulta más difícil de defender en esa 
postura, o el hecho de no conocer con profundidad la perspectiva de la permacultura hace que pierda fuerza ante los argumentos que sostienen los alumnos que deciden la agroecología. Otros grupos permanecen sin cambios tras la discusión en pequeño grupo.

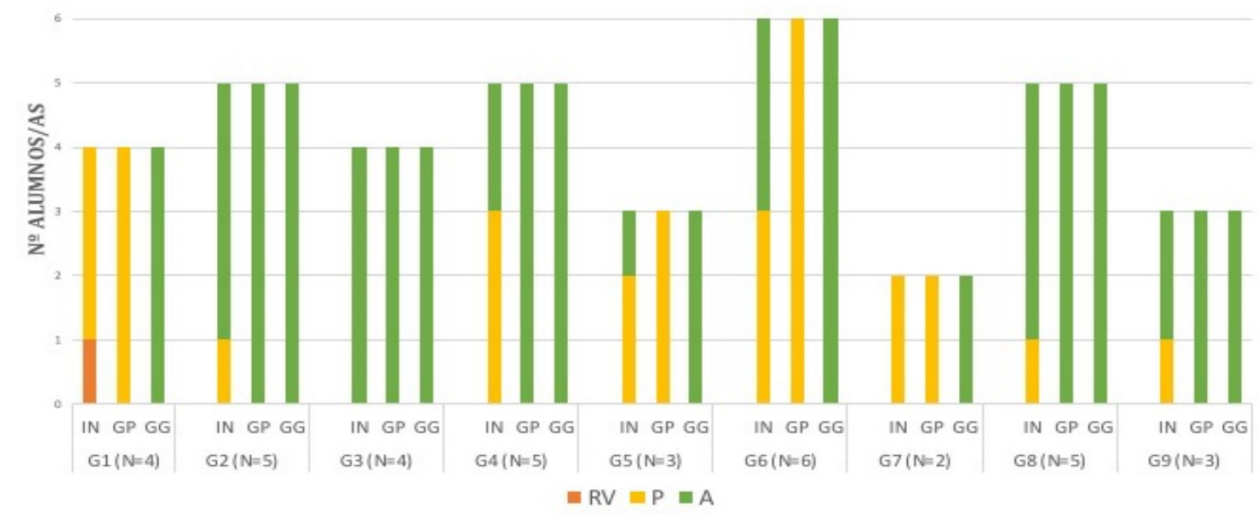

Figura 3. Opinión del alumnado sobre el marco de referencia (RV: revolución verde; P: Permacultura y A: Agroecología) desde el que diseñar el huerto a lo largo de la propuesta didáctica: I (Individual), GP (Equipos de Trabajo) y GG (Puesta en común en grupo clase). (Fuente: elaboración propia).

\section{Actividad pequeño grupo}

De los nueve equipos de trabajo que conforman el grupo clase, seis decidieron diseñar el huerto ecológico desde la perspectiva agroecológica, el resto consideró la permacultura como mejor opción. En cuanto a los tres equipos que optaron por la permacultura, uno ofreció argumentos que podrían considerarse como poco adecuados dado que toman la idea de «dejar que el medio siga su curso» (Equipo 1), cuando el concepto es más bien imitar a la naturaleza. Si bien incluyen uno de los principios éticos básicos de la permacultura como es cuidar la tierra, están ausentes los otros dos (el cuidado de la gente y compartir los recursos). Comentan además la idea de gestionar los recursos para beneficio propio, sin contemplar la idea de beneficio mutuo (trabajar con la naturaleza). Otro equipo aportó argumentos que ofrecieron dos ideas contrapuestas para apoyar dicho marco; por un lado, indican que la permacultura «busca las maneras y respuestas para que nuestras vidas sobre este planeta sean más sostenibles» (Equipo 5), en la que se mantiene una visión antropocéntrica entre la relación del hombre con el medio. Por otro, este grupo argumenta que la permacultura «concibe la restauración de medios naturales afectados al mismo tiempo que gestiona los recursos de manera sostenible para el beneficio mutuo de la especie humana y la naturaleza», una concepción más avanzada que la anterior. Finalmente, el tercer equipo ofreció argumentos adecuados, dado que expresan «la necesidad de utilizar los recursos de una manera sostenible», incluyendo la visión de incorporar técnicas específicas que pueden ser trasladadas al huerto (Equipo 7). Si bien no especifican qué tipo de relaciones se favorecen a través de esta perspectiva, o entre qué elementos se dan esas relaciones, sí las tienen en cuenta en sus respuestas.

Respecto a los seis equipos de trabajo que contemplaron la agroecología como perspectiva, se observa que algunos equipos emplean más argumentos en contra que 
a favor de dicha perspectiva a partir de los principios que sustenta la agricultura convencional. Probablemente por las diversas similitudes y coincidencias que encuentran entre agroecología y permacultura. Así, se registran respuestas del tipo «la agricultura convencional no la utilizaremos porque se emplean pesticidas, siendo perjudicial para el medioambiente» (Equipo 3). No obstante, el argumento más frecuente empleando por los grupos fue la idea de viabilidad a corto plazo, es decir, cinco de los seis equipos que eligieron la agroecología afirman que esta visión es más abarcable, principalmente porque «la permacultura necesita un compromiso social que requiere de mucho tiempo del que no disponemos» (Equipo 3), o bien argumentan que «hemos decidido elegir esta perspectiva porque es la que podemos llevar a cabo de forma más fácil en nuestro huerto» (Equipo 4), de forma muy similar a las ideas ofrecidas por los otros grupos, y muy coherente con lo analizado en las respuestas individuales en el apartado anterior. El equipo 3, además, alude explícitamente en su respuesta al objetivo productivo respecto al didáctico a la hora de emplear el huerto en la asignatura de EAI. Este aspecto debe ser tenido en cuenta en futuros cursos, porque, a pesar de que el huerto es considerado más como un recurso didáctico, para muchos alumnos es un aliciente obtener y poder recoger la cosecha. Para ello, una alternativa sería emplear semillas y plantas con ciclos de vida más cortos, poder cerrar procesos y llegar a obtener nuestras propias semillas ganando en autonomía y reduciendo entradas al sistema.

\section{Actividad gran grupo}

Al realizar la puesta en común en la que los grupos compartieron sus decisiones, se observó que ninguno de ellos optó por la agricultura convencional. En general, el argumento más frecuente fue el hecho de usar productos químicos, pero se explicitaron otras ideas como el uso de monocultivo y el impacto hacia el medio como aspectos incongruentes con el diseño de un huerto ecológico. Por tanto, se consensúa con el grupo clase la existencia de dos modelos que permiten alcanzar nuestro objetivo.

Los grupos que defendían la agroecología emplearon un argumento decisivo que supuso que el grupo clase optara finalmente por este modelo frente a la permacultura. Los equipos aludían a la idea de que «la permacultura abarca mucho más, pensamos que la agroecología puede ser mejor para nuestro huerto al ajustarse mejor a las necesidades que ahora tenemos» (Equipo 8). Esto hizo que existiera un consenso generalizado en el grupo clase ante dicha perspectiva. A la hora de profundizar en los tres marcos, la docente presentó la permacultura como una perspectiva holística y aplicable a todos los ámbitos de nuestra vida, utilizando para ello el recurso la flor de la permacultura, que expone 7 dominios de acción. Esta información probablemente hizo que parte del alumnado considerara más compleja la aplicación de esta perspectiva al huerto, lo cual pudo condicionar su decisión.

Por tanto, aunque la decisión final fue diseñar nuestro huerto desde la agroecología, se llegó al acuerdo de que ambas perspectivas estaban muy interrelacionadas, de hecho la permacultura se nutre de la agroecología, pero también de otros campos como la economía, el desarrollo comunitario o la bioconstrucción, entre otros. La permacultura se visualizó como una filosofía de vida, para algunos alumnos inalcanzable, tanto en el huerto como al día a día, debido a los estilos de vida y al sistema capitalista imperante actualmente. No obstante, de acuerdo a Bello et al. 
(2010), la agroecología también supone una manera de vivir, no sólo de producir o de comercializar, supone una manera de crear y de hacer. Sin duda son perspectivas, ambas complejas, que requieren de un mayor análisis y profundidad.

Finalmente, como consecuencia de la perspectiva consensuada, los equipos de trabajo expusieron sus acciones (tabla 3). Se pudo constatar que algunas de ellas eran similares desde ambas perspectivas, lo que refuerza la idea de las conexiones y relaciones que conviven entre la agroecología y la permacultura, y remarca la idea fuerza de que ambas no son excluyentes, sino complementarias.

Tabla 3. Acciones propuestas por los equipos de trabajo para llevar a cabo en el huerto según la perspectiva escogida (Fuente: elaboración propia).

\begin{tabular}{ll}
\hline \multicolumn{1}{c}{ Acciones propuestas por los 9 equipos de trabajo } & $\mathrm{N}^{\circ}$ (\% frecuencia) \\
\hline Trabajar de forma cooperativa & $(1) 8,3$ \\
Respetar el Medio Ambiente & $(1) 8,3$ \\
No usar pesticidas ni productos químicos & $(1) 8,3$ \\
Aprovechar el agua de lluvia & $(3) 25,0$ \\
Aprovechar recursos eficazmente & $(1) 8,3$ \\
Rotación de cultivos & $(1) 8,3$ \\
Asociar cultivos & $(1) 8,3$ \\
$\begin{array}{l}\text { Cuidar el suelo (lo vivo y no vivo) con prácticas } \\
\text { de laboreo inofensivas }\end{array}$ & $(1) 8,3$ \\
Usar abonos de lombriz & $(1) 8,3$ \\
$\begin{array}{l}\text { Elaborar nuestros propios productos para } \\
\text { combatir plagas y enfermedades de las plantas }\end{array}$ & $(1) 8,3$ \\
Aumentar la diversidad de especies vegetales & \\
atendiendo a la época del año & $(1) 8,3$ \\
\hline
\end{tabular}

\section{Conclusiones y reflexiones finales}

Desde la asignatura de EAI se ha querido mostrar dos perspectivas consideradas como alternativas para caminar hacia sistemas de producción más sostenibles respecto al modelo de agricultura convencional. Tanto la permacultura como la agroecología son consideradas idóneas como modelos para trabajar desde los huertos universitarios. Si bien, la perspectiva agroecológica se ha optado como adecuada dada su transposición didáctica a la enseñanza, a través de la agroecología escolar. Esta implica una práctica educativa que propone la transformación del sistema alimentario escolar en sus diferentes ámbitos (producción, transformación y consumo), a través del uso de los huertos ecológicos escolares, la cocina y los comedores escolares, mediante la participación de toda la comunidad educativa (Llerena, 2015). Se considera un marco de referencia idóneo para formar a los futuros docentes, con objeto de trasladar dichos aprendizajes a su futuro profesional. En esta línea, existen diversas iniciativas impulsadas desde otras universidades españolas, no sólo desde la formación inicial del profesorado, sino también desde otras titulaciones académicas, que contemplan la agroecología como un escenario sobre el que realizar prácticas, investigar, experimentar, reflexionar y actuar, de manera conjunta, entre comunidad universitaria y ciudadanía (Eugenio y Aragón, 2016).

Por otro lado, la propuesta didáctica diseñada ha permitido abarcar los objetivos anteriormente expuestos, que son: formar a futuros docentes más conscientes de sus modelos sobre la producción de alimentos, al plantearse de dónde procede lo que comemos y el impacto ambiental que supone cualquier actividad agrícola. Asimismo, se contribuye aproximar a los alumnos a distintos modelos alternativos de 
producción y posicionarse en un marco de referencia que les permita dar sentido a las acciones que puedan llevar a cabo en un futuro profesional, o bien en su vida diaria la hora de consumir productos procedentes de un modelo u otro. Además, favorece el sentido crítico del alumnado y su empoderamiento; para muchos, es muy evidente que no se deben utilizar productos químicos sintéticos para producir alimentos, sin embargo, pocas veces se reflexiona sobre las numerosas consecuencias ambientales que esto conlleva. Asimismo, pocas veces son capaces de proponer alternativas que permitan avanzar hacia el cambio y actuar ante los diversos problemas socioambientales. Tras la propuesta, los estudiantes son más conscientes del poder que posee el consumidor dentro del sistema alimentario y las desigualdades sociales que conlleva la agricultura convencional, así como sus consecuencias medioambientales.

Finalmente, al proporcionarles un marco de referencia alternativo, en este caso, desde la agroecología, cobra mayor sentido muchas de las acciones que suelen llevarse a cabo en el huerto ecológico, traspasando una idea simplista, porque el docente las considere oportunas o pertinentes, para asumir que existe todo un marco teórico que las sustenta.

Como reflexión final, se espera que el diseño de propuestas didácticas de este tipo contribuya a la formación de futuros educadores que valoren el potencial didáctico que posee el huerto escolare como herramienta para el cambio, que permita, como exponen Rodríguez-Marín, Fernández-Aguayo y García (2015), transcender al ámbito educativo la capacidad de investigar y resolver los problemas socioambientales, más allá de gestores y expertos. Se espera poder continuar con esta línea de trabajo, en la que se contemplen aspectos relevantes desde la didáctica de las ciencias, como son el diseño de propuestas basadas en los modelos explicativos del alumnado, los aprendizajes en contexto en torno a temas relevantes en el día a día del alumnado, como es el consumo de nuestros alimento a través de los huertos universitarios, y que implique el desarrollo de destrezas como la argumentación, tan implicadas en la competencia científica.

\section{Agradecimientos}

Agradecemos al Programa de Huertos Escolares de la Diputación de Cádiz por el material y apoyo técnico facilitado cada año. Al Decanato de la Facultad de Ciencias de la Educación por ceder el espacio cada curso académico y al Departamento de Didáctica de la Universidad de Cádiz por apoyar esta iniciativa.

\section{Referencias bibliográficas}

Altieri, M. (2013). La agroecología frente a la crisis alimentaria global. Letras Verdes. Revista Latinoamericana de Estudios Socioambientales, 4, 3-4.

Altieri, M. y Nicholls, C. (2000). Agroecología. Teoría y práctica para una agricultura sustentable. México: PNUMA.

Altieri, M. y Nicholls, C. I. (2017). Conversión agroecológica de sistemas convencionales de producción: teoría, estrategias y evaluación. Ecosistemas, 16(1), 3-12. 
Amat, A. (2013). Traspasando barreras: construyendo un proyecto comunitario a partir del huerto escolar. Enseñanza de las Ciencias, número extraordinario, 149-153.

Aragón, L. (2017). Evaluando el uso del huerto ecológico en la formación inicial de maestros/as desde la asignatura de Educación Ambiental en Infantil. Enseñanza de las Ciencias, número extraordinario, 779-785.

Aragón, L., Jiménez-Tenorio, N., y Oliva, J. M. (2015). El huerto ecológico universitario como herramienta para el estudio de problemas ambientales: una propuesta educativa en el grado de educación primaria. En AIDIPE (Ed.), Investigar con y para la sociedad (Vol.3, pp. 1849-1854). España: Bubok.

Aragón, L., y Cruz, I. M. (2016a). Del Huerto Ecológico Universitario al aula de infantil: experiencias educativas en torno a problemas ambientales en la etapa de infantil. Revista Internacional de Educación Preescolar e Infantil, 2(1), 40-48.

Aragón, L., y Cruz, I. M. (2016b). ¿Cómo es el suelo de nuestro huerto? El Aprendizaje Basado en Problemas como estrategia en Educación Ambiental. Una experiencia desde el Grado de Maestro/a en Educación Infantil. Didáctica de las Ciencias Experimentales y Sociales, 30, 171-188.

Aragón, L., y Rodríguez-Marín, F. (2019, prensa). ¿Qué percepciones iniciales tiene el profesorado en formación inicial sobre el uso en infantil de Huertos Ecodidácticos? En Actas VI Congreso Internacional de Docencia Universitaria, Vigo: CINDU.

Barrera, A. (2011). Nuevas realidades, nuevos paradigmas: La Nueva Revolución Agrícola. Comunica online. 8, 10-21.

Barrón, Á., y Muñoz, J.M. (2015). Los huertos escolares comunitarios: fraguando espacios socioeducativos en y para la sostenibilidad. Foro de Educación, 13(19), 213-239.

Bello, A., Jordá, C., y Tello, J. C. (2010). Agroecología y producción ecológica. Madrid: Consejo Superior de Investigaciones Científicas.

Caballero, A. y Montes, J. (1997). Agricultura sostenible. Un acercamiento a la permacultura. México DF: Servicios Educativos y de Investigación Social, A.C.

Ceballos, M. (2017). Aprovechamiento didáctico de los huertos escolares en centros de Sevilla. Enseñanza de las Ciencias, número extraordinario, 787-792.

Ceballos, M., Escobar, T. y Vílchez, J. E. (2014). El huerto escolar: percepción de los futuros maestros sobre su utilidad didáctica. En APICE (Comp.), 26 Encuentros de Didáctica de las Ciencias Experimentales y segunda Escuela de Doctorado, (pp. 285-292). Huelva: Universidad de Huelva.

Ceccon, E. (2008). La revolución verde tragedia en dos actos. Ciencias, 1(91), 21-29.

Espluga, J. (2001). Percepción del riesgo y uso de pesticidas en la agricultura. Ecología política, 22, 17-30. 
Estrella, A. (2018). Los Huertos escolares. Avance del mapeo realizado previo al I Encuentro Estatal de Huertos Escolares. En Memoria del I Encuentro de Huertos Escolares, Tudela (Navarra).

Estrella, A., Del Valle, J. y Jiménez, L. (2017). Diagnóstico de la Red de Huertos Escolares Sostenibles del Ayuntamiento de Madrid. Madrid: Ayuntamiento de Madrid.

Eugenio, M. (2016). Huerto ecológico como recurso y contexto para la enseñanzaaprendizaje de las Ciencias Naturales en la formación inicial de maestros/as de Infantil. Huertos ecodidácticos. Compartiendo experiencias educativas en torno a huertos ecológicos. Actas del I Encuentro de Huertos Ecodidácticos. Soria: Jolube.

Eugenio, M., y Aragón, L. (2016). Experiencias educativas en relación a la agroecología en la educación superior española contemporánea: presentación de la Red Universidades Cultivadas (RUC). Agroecología, 11(1), 31-39.

Eugenio, M., Zuazagoitia, D. y Ruiz-González, A. (2018). Huertos EcoDidácticos y Educación para la Sostenibilidad. Experiencias educativas para el desarrollo de competencias del profesorado en formación inicial. Revista Eureka sobre Enseñanza y Divulgación de las Ciencias, 15(1), 1501.

Holmgren, D. (2013). Permacultura: principios y senderos más allá de la sustentabilidad. Castellón: Argentina Kaicron.

García, A., Laurín, M., Llosá, M. J., Gonzálvez, V., Sanz, M. J., y Porcuna, J. L. (2006). Contribución de la agricultura ecológica a la mitigación del cambio climático en comparación con la agricultura convencional. Agroecología , 1, 75-88.

Gómez, L. F., Ríos-Osorio, L. y Eschenhagen, M.L. (2015). Las bases epistemológicas de la agroecología. Agrociencia, 49(6), 679-688.

Gónzalez, J. (1984). Innovación tecnológica en la agricultura y acumulación de capital un análisis crítico de la revolución verde. Revista de Estudios Agrosociales, 142, 7-30.

Griffon, D. (2008). Estimación de la biodiversidad en Agroecología. Agroecología, 3, 25-31.

Kaufman, M. (1995). Huertos y más huertos. Investigando en la escuela infantil. Investigación en la Escuela, 25, 87-100.

Laurín, M., Llosá, M. J., Gonzálvez, V., y Porcuna, J. L. (2006). El papel de la agricultura ecológica en la disminución del uso de fertilizantes y productos fitosanitarios químicos. En Actas VII Congreso SEAE de Agricultura y Alimentación Ecológica. Zaragoza: SEAE.

Llerena, G. (2015). Fundamentación teórica y estudio de casos sobre el desarrollo de los huertos escolares con el referente de la agroecología. Tesis Doctoral, Universidad Autónoma de Barcelona.

Llerena, G., y Espinet, M. (2017). Agroecología escolar. Barcelona; Pollen Edicions.

Martínez, R. (2002). Agroecología: atributos de sustentabilidad. Inter Sede, III(5), 2545. 
Martínez-Madrid, B. y Eugenio, M. (2016) Acercamiento a la Agroecología en la Infancia: propuestas educativas y reflexiones. Agroecología 11(1), 7-18.

Mollison, B. (1979). Permaculture A Designers' Manual. Australia: Tagari.

Rekondo, M., Espinet, M. y Llerena, G. (2015) La construcción discursiva de la competencia eco-ciudadana en la escuela: La realización de un diseño tecnológico colaborativo en agroecología escolar. Investigación en la Escuela, 86, 7-19.

Rodríguez, R. M., Hernández, R., Hernández, J. C. y Pérez, A. C. (2015). La permacultura, una alternativa en la producción de alimentos desde la escuela y la comunidad. Revista de Cooperativismo y Desarrollo, 4(1), 85-94.

Rodríguez-Marín, F., Fernández-Arroyo, J. y García, J. E. (2015). El huerto escolar ecológico como herramienta para la educación en y para el decrecimiento. Investigación en la Escuela, 88, 35-48

Rodríguez-Marín, F., Fernández-Arroyo, J., Puig-Gutiérrez, M., y García, J. E. (2017). Los huertos escolares ecológicos, un camino decrecentista hacia un mundo más justo. Enseñanza de las Ciencias, número extraordinario, 805-810.

Torres, P. y Soto, J. (2016). JAULAS ABIERTAS, una Común-Unidad de Aprendizaje en-Red-Dando a la Ciudadanía. En M. Eugenio y L. Aragón (Coords.), Huertos EcoDidácticos. Compartiendo experiencias educativas en torno a huertos ecológicos (pp. 21-25). Huesca: Jolube.

Universidad de Cádiz. (2012). Memoria del Título del Grado en Educación Infantil. 